\title{
3D-printing on textiles - an investigation on adhesion properties of the produced composite materials
}

\author{
Maryna Gorlachova ${ }^{1} \cdot$ Boris Mahltig ${ }^{1}$ (])
}

Received: 2 October 2020 / Accepted: 6 May 2021 / Published online: 11 May 2021

(c) The Author(s) 2021

\begin{abstract}
The actual paper is related to adhesive properties of 3D objects printed on cotton textile fabrics. For practical applications of 3D prints in the textile sector, the adhesion of the printed object on the textile substrate is an important issue. In the current study, two different types of polymers are printed on cotton - polylactide acid (PLA) and polyamide 6.6 (Nylon). Altogether six cotton fabrics differing in structure, weight and thickness are evaluated. Also, the effect of washing and enzymatic desizing is investigated. For printing parameters, best results are gained for elevated process temperatures, intermediate printing speed and low Z-distance between printing head and substrate. Also, a textile treatment by washing and desizing can improve the adhesion of an afterwards applied 3D print. The presented results are quite useful for future developments of 3D printing applications on textile substrates, e.g. to implement new decorative features or protective functions.
\end{abstract}

Keywords 3D-printing $\cdot$ PLA $\cdot$ Nylon $\cdot$ Cotton $\cdot$ Interpenetration

\section{Introduction}

3D-printing is a versatile tool to produce three-dimensional objects of individual and extraordinary geometry [1-3]. Usually the 3D objects are produced as single independent pieces, e.g. as unique prototypes. However, 3D printing can be done also on other substrates with the aim of modification of these substrates by the added 3D object [4-7]. This application purpose is similar to traditional printing techniques. However, traditionally mainly two-dimensional images are printed. In contrast, by $3 \mathrm{D}$ printing also objects can be applied which have a three-dimensional structure [8]. Textile materials are often used as substrate for printing processes. Also, the 3D-printing on textiles can be used to create useful applications, as e.g. printed buttons, decorative or protective elements $[4,9,10]$. 3D printing can be also used as tool for application of conductive layers and the fixation of electronic components on textile surfaces, which can find applications in smart textiles [11-14]. A very fascinating application in this field is the realization of printed

Boris Mahltig

Boris.mahltig@hs-niederrhein.de

Faculty of Textile and Clothing Technology, University of Applied Sciences Niederrhein, Webschulstr. 31, 41065 Mönchengladbach, Germany electroluminescent devices on textile materials [15]. 3D printing on textiles is also discussed as a first step to realize 4D textile materials [16]. These resulting materials can be named as 3D-prints on textiles but also as a kind of composite material, where two different types of materials are combined. For textile applications it is absolutely necessary that the 3D print adhere to the textile substrate. With this background, the aim of this current paper is to investigate parameters influencing the adhesion of 3D prints on cotton fabrics. Textiles from cotton are chosen, because cotton is in the textile sector the most used natural fiber. After polyester fibers, cotton is the second most used fiber globally.

Earlier studies are focusing on a broad field of various parameters influencing the success of the 3D printing on textile substrates. The precoating of fabrics from cotton, linen or polyester can enhance the adhesion to an afterwards applied 3D printed object $[17,18]$. However, this technique requires an additional coating process before $3 \mathrm{D}$ printing is performed, this can be of course a disadvantage for transfer to commercial applications. Also, the influence of distance between printing nozzle and textile substrate (Z-distance) is investigated [19]. A good overview on PLA 3D printed objects and their adhesion onto a big variety of different textiles made from cotton, polyester and acrylics is given by Mpofu et al. [20]. These authors use a reasonable regression method to compare a large amount of different textile 
Table 1 Overview on specifications of used filament materials

\begin{tabular}{lll}
\hline Property & PLA-filament & Nylon-filament \\
\hline Color & yellow & White \\
Filament diameter $[\mathrm{mm}]$ & 1.75 & 1.75 \\
Density $\left[\mathrm{g} / \mathrm{cm}^{3}\right]$ & 1.24 & 1.14 \\
Glass temperature $\mathrm{T}_{\mathrm{G}}\left[{ }^{\circ} \mathrm{C}\right)$ & 65 & 68 \\
Melting temperature $\mathrm{T}_{\mathrm{M}}\left[{ }^{\circ} \mathrm{C}\right]$ & 160 & 195 \\
Decomposition temperature $\mathrm{T}_{\mathrm{D}}\left[{ }^{\circ} \mathrm{C}\right]$ & 235 & 312 \\
Recommended temperature for extrusion $\left[{ }^{\circ} \mathrm{C}\right]$ & 190 to 210 & 228 to 235 \\
Recommended temperature for printing table $\left[{ }^{\circ} \mathrm{C}\right]$ & 40 to 50 & Circa 45 \\
Geometry of printed object - height/length/depth $[\mathrm{mm}]$ & $21 / 12 / 4$ & $21 / 16 / 6$ \\
\hline
\end{tabular}

materials. However, an effect of washing or any other kind of pretreatment is not in the scope of their investigations. An intensive investigation of PLA and Nylon 3D printed objects and their adhesion to different types of fabrics from synthetic fibers is reported by Sanatgar et al. [21].

In contrast to these earlier studies, the actual investigations are related to the comparison of differently structured cotton fabrics with 3D printed objects from PLA and Nylon. Beside the textile parameters also printing parameters are evaluated and optimized reasonably. A special view is done on the influence of washing procedures and enzymatic desizing. Recently, the short review of Kozior et al. summarizes main articles, findings and developments in the field of $3 \mathrm{D}$ printing on textiles with a special view on the adhesion properties of the 3D printed object [22]. In fact, due to the manifold types of materials, printers and techniques to measure the adhesion, it is quite difficult to unify the results from different research groups. Especially with the procedure of adhesion measurement and their reproducibility the main difficulty appears. Nevertheless, Kozior et al. give the interesting approach to translate basic physical processes to the printing parameters influencing the adhesion. He mentions especially the wetting, the diffusion and the pressure. An interesting approach is here also the after-treatment, by annealing or ultrasonic $[22,23]$.

\section{Experimental section}

\section{Materials}

As materials for 3D printing two different types of polymer filaments are used - a polylactide acid (PLA) filament and a polyamide 6.6 (Nylon) filament. The PLA filament (polylactic acid) is supplied by the company Filamentworld (Neu-Ulm, Germany). The used filament type is named "Sonnengelb" with a diameter $1.75 \mathrm{~mm}$. The Nylon filament (polyamide 6.6; PA 6.6) is supplied by the company Taulman3D (Indiana, USA). The used filament type is "Nylon-230-Filament" with a diameter of $1.75 \mathrm{~mm}$. Further properties of used filaments are summarized in Table 1. Especially mentioned are the process temperatures recommended by the different suppliers. The 3D printing is performed on six different types of cotton fabrics. These fabrics and related properties are summarized in Table 2. For all textile materials a washing and enzymatic desizing procedure is done as precleaning procedure before $3 \mathrm{D}$ printing. Following, the performance of cotton fabrics with and without this precleaning is compared. This washing procedure is done with $2.58 \mathrm{~kg}$ cotton fabric washed in one liter water at $40{ }^{\circ} \mathrm{C}$. An industrial washing machine IPSO ILG is used. As washing agent $16.5 \mathrm{~g} / \mathrm{L}$ Felosan FOX (CHT,

Table 2 Overview on the investigated cotton fabrics; shown are materials properties and parameters used in 3D printing on these fabrics. For material TEGEWA drop test results, the average values are given and the standard deviation is mentioned in brackets

\begin{tabular}{|c|c|c|c|c|c|c|c|c|}
\hline \multirow[t]{2}{*}{ No } & \multirow[t]{2}{*}{ Type } & \multicolumn{3}{|c|}{ Material properties } & \multicolumn{2}{|c|}{$\begin{array}{l}\text { TEGEWA drop test sink in } \\
\text { time [s] }\end{array}$} & \multicolumn{2}{|c|}{ Printing parameter } \\
\hline & & $\begin{array}{l}\text { Weight per } \\
\text { area }\left[\mathrm{g} / \mathrm{m}^{2}\right]\end{array}$ & Roughness $[\mu \mathrm{m}]$ & Thickness [mm] & Grey fabric & $\overline{\text { After washing }}$ & $\begin{array}{l}\text { Z-distance } \\
{[\mathrm{mm}]}\end{array}$ & $\begin{array}{l}\text { Com- } \\
\text { pression } \\
{[\mathrm{mm}]}\end{array}$ \\
\hline $\mathrm{F} 1$ & Plain weave & 112.8 & 0.04 & 0.2 & $>180$ & $13.6(2.37)$ & 0.2 & None \\
\hline $\mathrm{F} 2$ & Plain weave & 309.3 & 0.06 & 0.6 & $8.8(0.32)$ & $1.7(0.56)$ & 0.3 & 0.3 \\
\hline $\mathrm{F} 3$ & Twill weave & 306.8 & 0.11 & 0.48 & $3.0(0.15)$ & $1.2(0.26)$ & 0.3 & 0.18 \\
\hline $\mathrm{F} 4$ & Twill weave & 253.3 & 0.04 & 0.38 & $3.8(0.23)$ & $1.1(0.17)$ & 0.2 & 0.18 \\
\hline F5 & Knitwear, Single-jersey & 192.4 & 0.05 & 0.47 & $>180$ & $2.0(0.55)$ & 0.4 & 0.07 \\
\hline F6 & Knitwear, Single-jersey & 267.2 & 0.09 & 0.73 & $3.8(0.12)$ & $1.8(0.15)$ & 0.5 & 0.23 \\
\hline
\end{tabular}




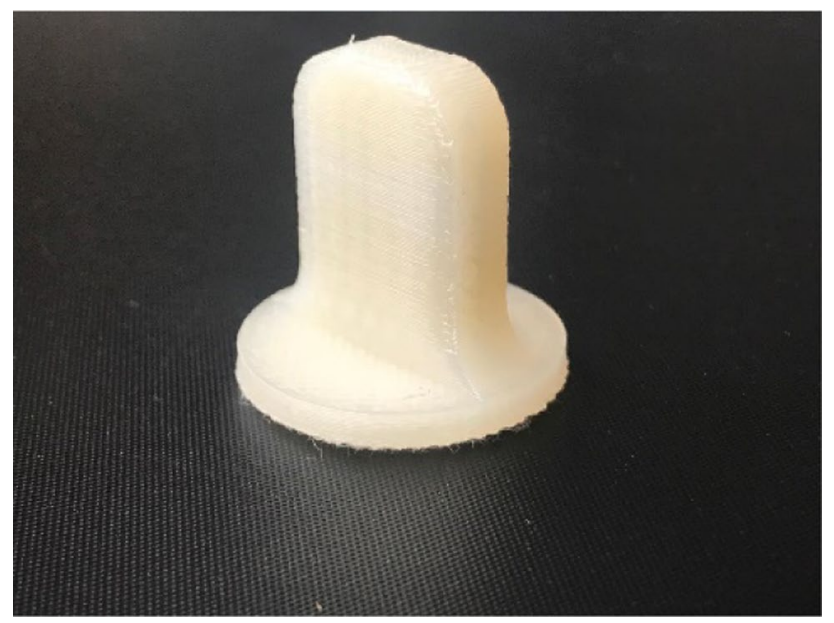

Fig. 1 3D printed object from Nylon prepared for application on textile fabrics for force measurements

Tübingen, Germany) is added. For enzymatic desizing the enzyme Beisol T 2090-G (CHT, Tübingen, Germany) is added in an amount of $25 \mathrm{~g}$ to the washing solution.

\section{Printing process}

All 3D objects are produced using a commercially available 3D printer Orcabot XXL Pro supplied by the company Prodim International BV (Helmond, The Netherlands). The printed objects are prepared for the following force measurements. A picture of a 3D printed object used in current investigations is shown as example in Fig. 1. The weight of these objects is $3 \mathrm{~g}$ for both types of used filament. Also, the height is with $21 \mathrm{~mm}$ similar for both filaments. However, length and depth are different for PLA and Nylon material (see values given in Table 1). The used parameters for printing are different for both types of filaments and are summarized in Table 3. For first evaluation of printing parameters, orientation experiments are done (discussed

Table 3 Overview on used printing parameters

\begin{tabular}{|c|c|c|c|c|c|}
\hline \multirow[t]{2}{*}{ Filament } & \multirow[t]{2}{*}{ Event } & \multicolumn{2}{|c|}{$\begin{array}{l}\text { Process temperatures } \\
{\left[{ }^{\circ} \mathrm{C}\right]}\end{array}$} & \multicolumn{2}{|c|}{$\begin{array}{l}\text { Printing speed }[\mathrm{m} / \\
\text { min] }\end{array}$} \\
\hline & & $\begin{array}{l}\text { Extrusion } \\
\text { / filament }\end{array}$ & $\begin{array}{l}\text { Printing } \\
\text { table }\end{array}$ & General & $\begin{array}{l}\text { For first } \\
\text { printed } \\
\text { layer }\end{array}$ \\
\hline \multirow[t]{2}{*}{ PLA } & Orientation & 200 & 60 & 3.3 & 1.32 \\
\hline & $\begin{array}{l}\text { Compari- } \\
\text { son }\end{array}$ & 220 & 60 & 3.3 & 0.66 \\
\hline \multirow[t]{2}{*}{ Nylon } & Orientation & 230 & 90 & 2.0 & 0.8 \\
\hline & $\begin{array}{l}\text { Compari- } \\
\text { son }\end{array}$ & 250 & 90 & 2.0 & 1.0 \\
\hline
\end{tabular}

in Orientation according to printing parameters). As result from these orientation experiments the parameters for the final comparison of different cotton fabrics are optimized (discussed in Comparison of textile substrates). The used z-distances in the final experiments differ for the different cotton fabrics. The values are listed in Table 2 .

\section{Analytical methods}

The weight per area of used cotton fabrics are determined according to standard DIN EN 12,127, while the thickness of the fabrics is measured according to standard DIN EN ISO 5084. The surface roughness of cotton fabrics is determined using a 3D laser scanning microscope VK-X100 supplied by the company Keyence. Microscopic images of sample cross-sections are performed using a digital light microscope VHX-600 supplied by the company Keyence. The hydrophilic properties of the cotton fabrics are determined by using the TEGEWA drop test $[24,25]$. Here a drop of water is placed in a reproducible way on the textile and the time is measured until this drop sinks into the textile completely. A lower sink in time stands for a higher hydrophilic property of the textile. If a drop sinks not in during $3 \mathrm{~min}$, the measurement is stopped and a sink in time of $>180 \mathrm{~s}$ is recorded. The TEGEWA drop test is repeated three times with each sample and the average value is calculated and given together with the standard deviation.

Different testing arrangements are possible to determine the adhesion between a 3D printed object and a textile substrate [26]. Malengier et al. compared three different test arrangements - a perpendicular (vertical) test, a sheer test and a peel test. Finally, these researchers conclude that all these three tests can be used to determine adhesion of 3D-prints on textiles in a suitable way [26]. In the actual study a perpendicular tensile test is used. Disadvantageous for this test arrangement is the more time intensive production of testing samples due to a larger size of prepared 3D printed objects. An example of a printed testing object is shown in Fig. 1. These objects are printed on textile substrates with a size of $8 \mathrm{~cm} \mathrm{X} 20 \mathrm{~cm}$. The adhesion is tested by using force measurements during separation with a machine Zwick 1455 (Zwick/Roell GmbH, Ulm, Germany). Before measurement, all sample are maintained in standard conditions $\left(21^{\circ} \mathrm{C} ; 65 \%\right.$ humidity) for one day. For measurement, textile and 3D object are placed in the device as shown in Fig. 2. The force measurement is recorded as shown in Fig. 3. All measurements are repeated with three independently and similarly prepared 3D printed objects. The maximum force determined in this separation experiment is recorded and set as adhesion between the 3D printed object and the textile substrate. Finally, the average of the three measurements is calculated and shown in following figures as adhesion. The error bars shown in the graph are related to the differences between the performed three measurements. 


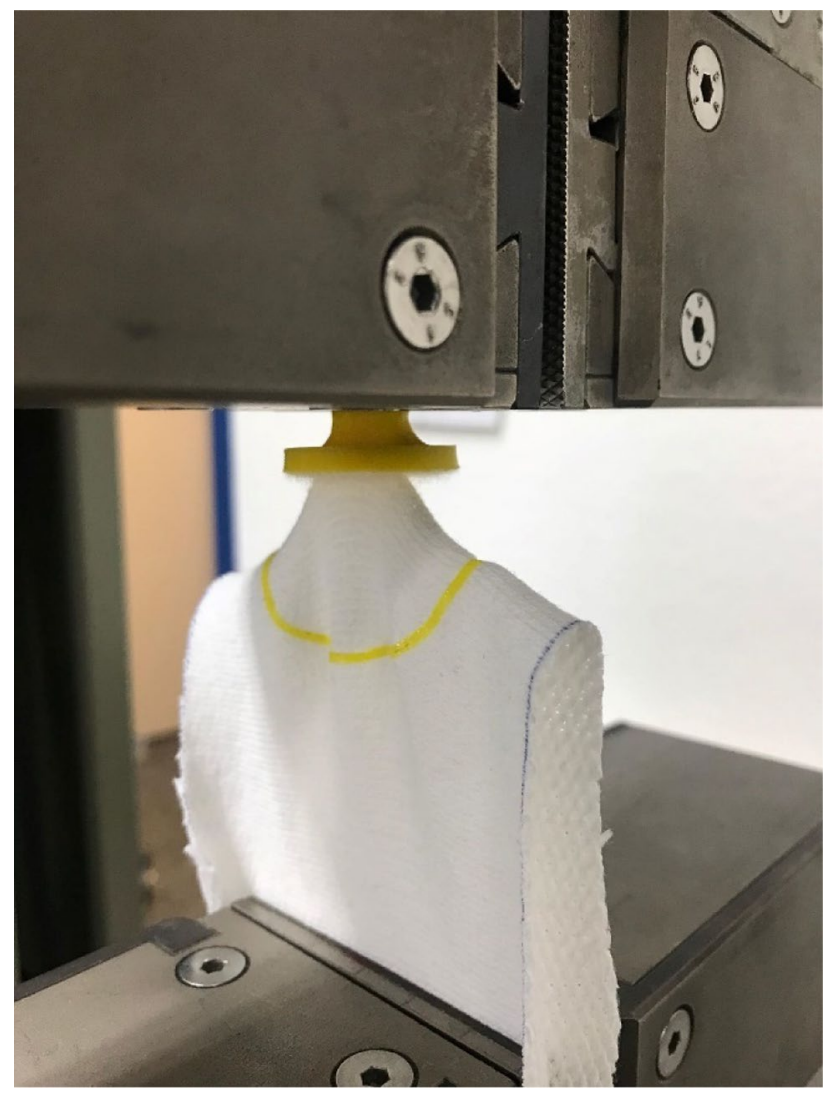

Fig. 2 Photograph of a sample during the separation test

\section{Results and discussion}

This section is divided in two parts. In the first part, orienting experiments are done to evaluate for each printing

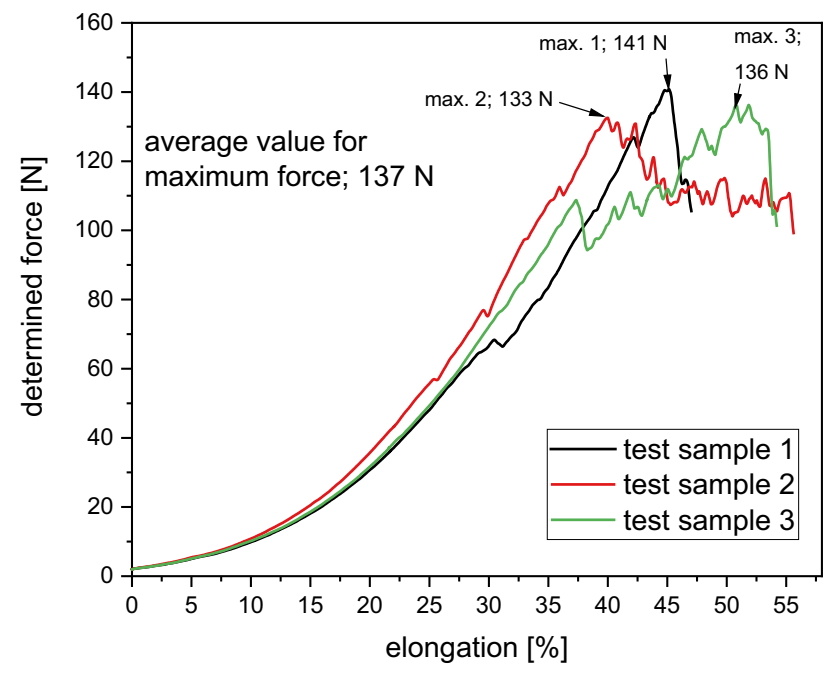

Fig. 3 Diagram of force measurements during separation of 3D-printed object (PLA) from a cotton fabric F6. Shown are the results of the three independently performed measurements parameter an optimal setting. For these orienting experiments only two different types of fabrics are used, the woven fabric F2 and the knitted fabric F6. In the second part, the printing parameters are set based on evaluation in the first part and the application on all six different types of cotton fabrics is investigated. Here, the optimal properties for the substrate parameters are evaluated.

\section{Orientation according to printing parameters}

Evaluated are here four different parameters - the Z-distance between printing head and printing table, the printing speed of first applied layer, the extrusion temperature and the temperature of the printing table.

For experiments with PLA-filament, the Z-distance is set to $0.7 \mathrm{~mm}$ and for Nylon-filament this Z-distance is set to $0.5 \mathrm{~mm}$. The other parameters for the orienting experiments are given in Table 3. During following experiments, three of these four parameters are set fixed and one parameter is modified in a range, which is reasonable according to the recommendation of the suppliers of filaments and 3D-printer. These orienting experiments are done with the PLA-filament on the cotton fabric F6 which is chosen as example for a knitted fabric. Experiments with the Nylon filament are done on the cotton fabric F2 which is chosen as an example for a woven fabric. Later in Comparison of textile substrates, both types of fabrics are used with both different types of printing filament and are compared with all six different types of cotton fabrics investigated. The evaluation to find an optimal Z-distance is done for printing with PLA-filament (Fig. 4). The distance between the printing head and the printing table (Z-distance) has a significant influence on the adhesion of the PLA specimen. In case of

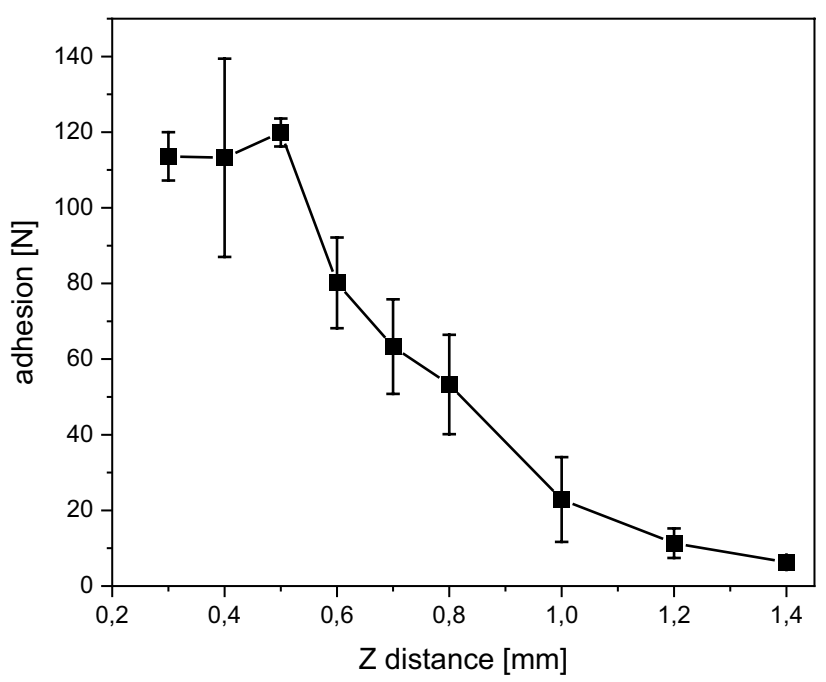

Fig. 4 Adhesion of PLA-print on cotton fabric (F6) print as function of $\mathrm{Z}$ distance, distance between printing head and ground 
low Z-distance of 0.2 to $0.5 \mathrm{~mm}$ high adhesion values of around $115 \mathrm{~N}$ are observed (Fig. 4). This range is determined by a high plateau level. From this plateau level the adhesion decreases continuously with increasing Z-distance. Due to the thickness of the cotton fabric F6 of $0.73 \mathrm{~mm}$, with low Z-distance the fabric is compressed by the printing head during the application of first printed layer. By this compression, the PLA polymer is directly feed into the fabric structure. The microscopic images shown in Fig. 5 give a good view, how the Z-distance influences the interpenetration of the cotton fabric by the printed PLA. The yellow colored PLA is clearly distinguished by the white cotton fabric. For the shown samples, two PLA layers are printed on the cotton and a cross-section is made. The printing in two different Z-distances $(0.5 \mathrm{~mm}$ and $0.8 \mathrm{~mm})$ is compared. It is clearly seen, that the smaller Z-distance leads to a stronger interpenetration of the coated cotton fabric by the PLA print. An earlier study investigating the influence on the Z-distance intensively reports the same decrease in adhesion as function of an increasing Z-distance [19]. However, this earlier study does not show a clear plateau value for small Z-distances. Nevertheless, the finding of a plateau value is reasonable, if a maximum interpenetration of the cotton by the printed PLA can be assumed. In that case, lower Z-distance cannot improve the interpenetration further. Based on these results, the Z-distance is set to low values during following experiments presented in Comparison of textile substrates. The chosen Z-distance depends on the thickness of the different cotton fabrics (Table 2). Nevertheless, the set-up of the printer allows only a minimum Z-distance of $0.2 \mathrm{~mm}$.

By increasing the printing-speed for the first applied layer, the adhesion decreases in a nearly linear matter (Fig. 6). Compared to the influence of the Z-distance, the effect of the printing speed on the adhesion is weak. It is also weak compared to the later discussed process parameters. However, it should be clear that the printing speed of the first

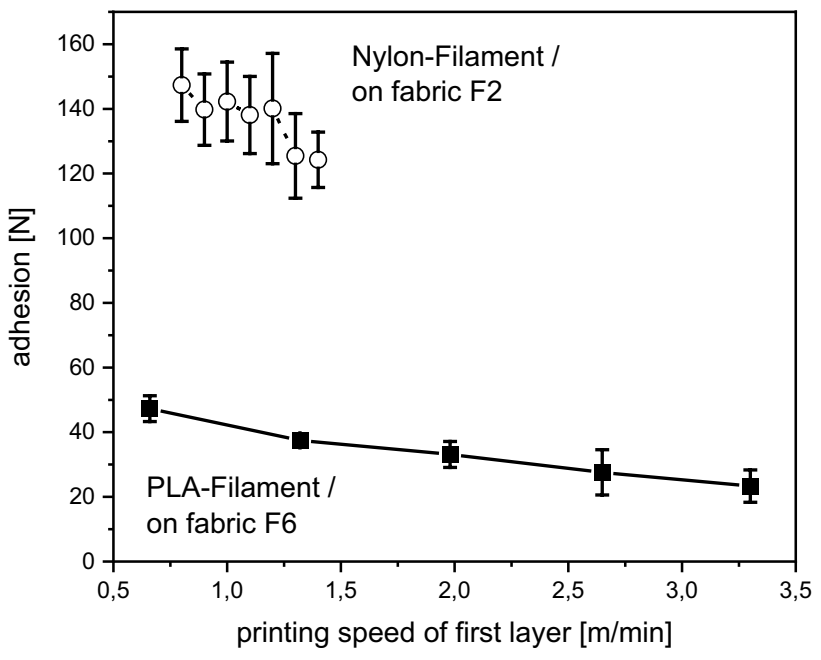

Fig. 6 Adhesion of print on cotton fabric (F6 and F2) as function of printing speed of the first layer. Compared are prints from two different filaments-PLA and Nylon

layer should be moderately slow. For following comparison experiments this printing speed is therefore set to $1.0 \mathrm{~m} /$ min for the Nylon-filament and $0.8 \mathrm{~m} / \mathrm{min}$ for printing with PLA-filament.

The effects of two different process temperatures are evaluated - the temperature of the printing table (printing ground) and the temperature of the printed filament (extrusion temperature). The temperatures chosen for current investigations are according to the recommend process temperatures of the suppliers (Table 1). Due to these recommendations, for the investigated Nylon filament, temperatures in a higher range are evaluated (Figs. 7 and 8). There is a clear correlation for both process temperatures observed. The adhesion is increasing with increasing temperature. However, the effect of the filament temperature is more significant compared to the temperature of printing table
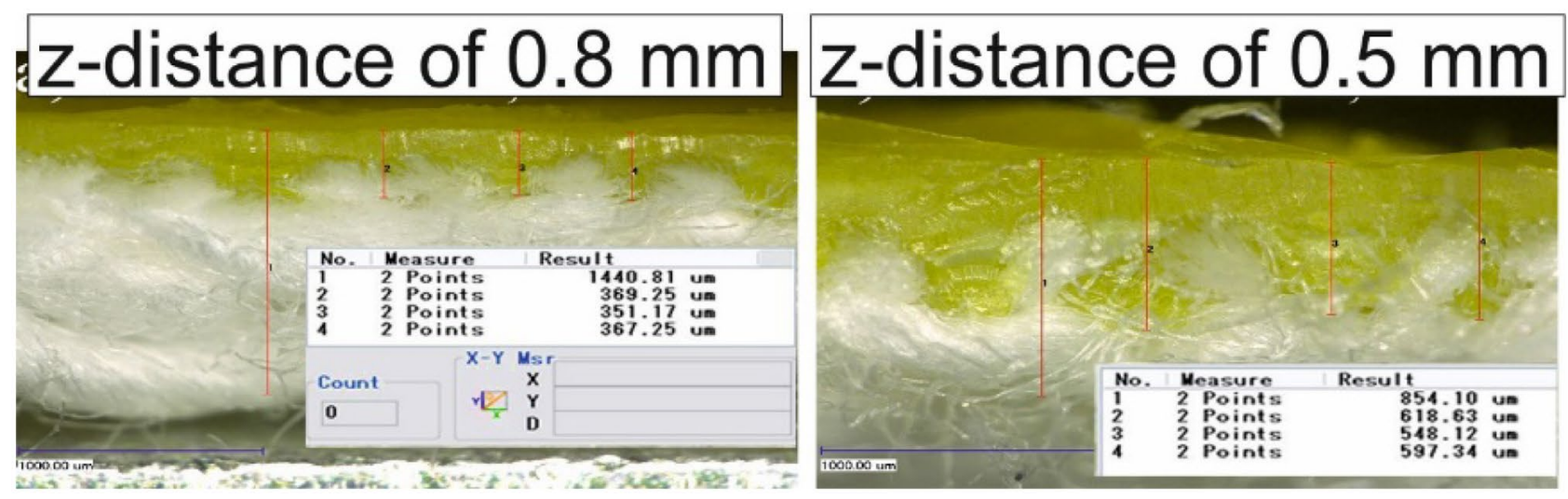

Fig. 5 Light microscopic images of cross-sections of PLA printed layers (two layers) on the cotton fabric F6. Compared are the applications performed with two different Z-distances (left image: $0.8 \mathrm{~mm}$ - right image: $0.5 \mathrm{~mm}$ ) 


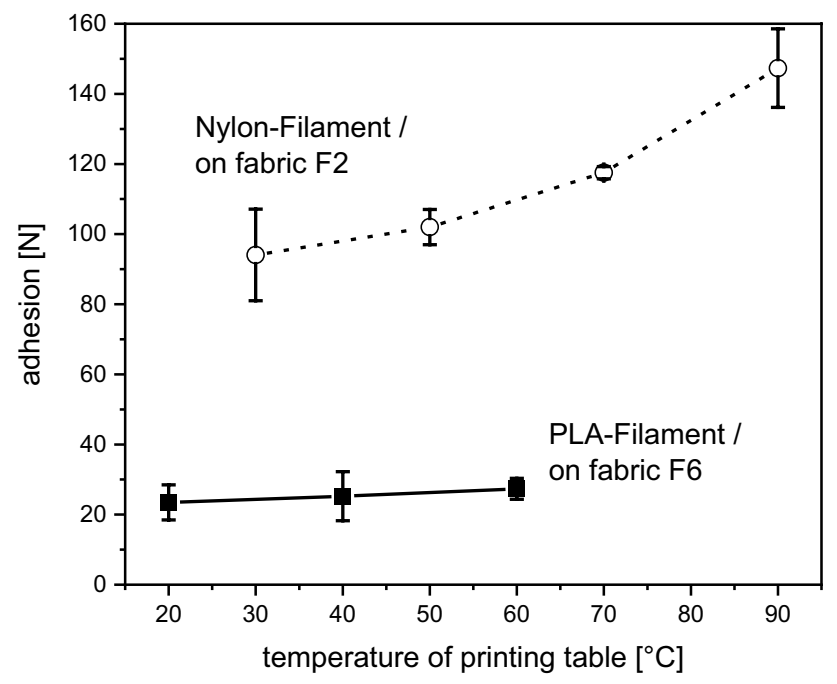

Fig. 7 Adhesion of print on cotton fabric (F6 and F2) as function of the temperature of the printing table. Compared are prints from two different filaments-PLA and Nylon

(Figs. 7 and 8). It can be assumed that higher temperatures lead to a more fluid polymer melt which is able to penetrate the treated cotton fabric more deeply, so the interpenetration is improved [21]. However, due to the thermal stability of the filament polymers, the extrusion temperature cannot be elevated unlimited. For the following comparative experiments, following extrusion temperatures are chosen $-220{ }^{\circ} \mathrm{C}$ for PLA- and $250^{\circ} \mathrm{C}$ for Nylon-filament. These temperature settings are higher compared to the process recommendations of the filament suppliers but still significantly below the given decomposition temperature.

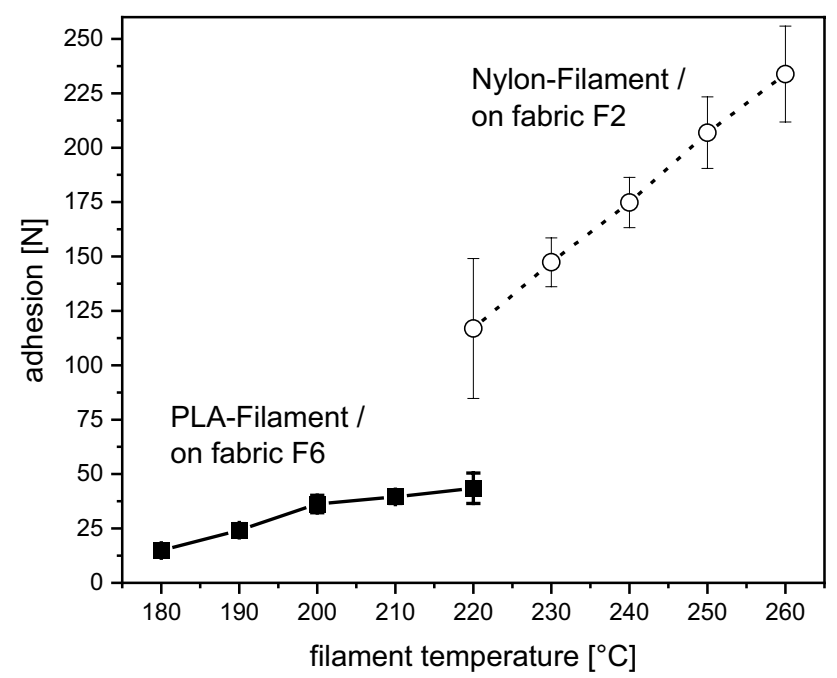

Fig. 8 Adhesion of print on cotton fabric (F6 and F2) as function of the filament temperature. Compared are prints from two different filaments-PLA and Nylon

\section{Comparison of textile substrates}

The experiments on six different cotton fabrics are done with the same printing parameters. Properties of the textile substrates are reported in Table 2. The used printing parameters are shown in Table 3. These printing parameters are chosen as result of the above described orientating experiments. Only the Z-distance is set individually for each type of cotton fabric depending on its fabric thickness (Table 2). Actually, the aim is to evaluate, which textile structure and properties enhance the adhesion of a 3D printed object on a textile surface. Beside the grey fabrics, also the printing results on washed cotton fabrics are evaluated. A summary of adhesion of 3D-objects on the different cotton fabrics is shown in Fig. 9. In this figure also, the printing results form PLA- and Nylon-filaments are compared.

Figure 10 shows photographs from PLA objects after their separation from different cotton fabrics during the force measurements. In summary, two different cases have to be distinguished. In the first case, there is a clear separation of the PLA object from the cotton fabric, without visible damage of the cotton fabric. Here, the main force for separation can be clearly assigned to the adhesion between the PLA object and the cotton substrate. In the second case, there is additional to this separation also a destruction of the cotton fabric by the applied mechanical force. Here, the measured force for separation is related to two different material properties - the adhesion of the 3D object to the cotton substrate and the mechanical stability of this cotton fabric. Especially for the fabrics F5 and F6, the delamination of the textiles happens only partly and the textile is damaged, so no further delamination occurs. For this case, the maximum separation

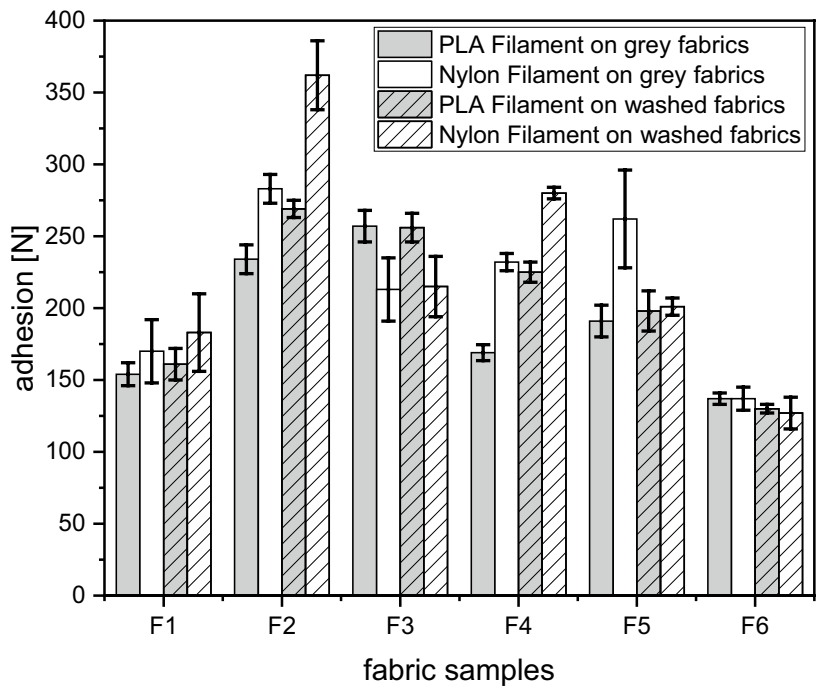

Fig. 9 Comparison of adhesion of 3D-printed objects placed on six different cotton fabrics (grey fabric and after a washing). Compared are prints from two different filaments-PLA and Nylon 
force cannot be assigned simply to the adhesion alone. It is instead a measurement of the mechanical stability of the fabric, which is obviously lower than the adhesion of this fabric to the printed PLA object. It could be estimated that a measurement with a textile of higher mechanical stability lead to higher adhesion values determined, so the adhesion of 3D object to the textile substrate is higher than the measured one. However, to set the measured adhesion in relation to the detached area and to present normalized data could lead to misleading numbers, because the measured force is not only related to the adhesion between 3D object and textile but also from the force needed for textile destruction.

By view on Fig. 10, only for the fabrics F1, F2 and F4 a separation of the PLA objects from cotton without damaging of the cotton is observed. Only for these three fabrics, the discussion of the adhesion properties is senseful.

The determined adhesion for these samples is increased with the thickness of the textile substrate and the weight per area of the textile substrate (Figs. 12 and 13). This result can be explained by the determined adhesion as function of the Z-distance between printing head and printing table, as earlier discussed in Orientation according to printing parameters. The fabric F1 has the smallest thickness of only $0.2 \mathrm{~mm}$ and the minimum Z-distance possible by the printer is also $0.2 \mathrm{~mm}$. In this case the printed filament is simply laid on the textile fabric. In case of the fabric F2 with the bigger thickness of $0.6 \mathrm{~mm}$ the Z-distance during the printing is set to $0.3 \mathrm{~mm}$, so the fabric is compressed during the printing with $0.3 \mathrm{~mm}$. By this compression the printed filament is forced into the textile structure and not simply laid down on the textile surface. Please, compare here also Fig. 5. The stronger compression of thicker textile substrates during printing leads to a stronger interpenetration of the coated cotton fabric by the PLA print. An earlier investigation reported on this issue by view on different Z-distances while printing on the same type of textile lead to similar results [19].
For all these three fabrics (F1, F2 and F4), an increase in adhesion is determined if the fabrics are washed before the 3D print is applied (Figs. 12 and 13). By washing, impurities are removed from the cotton surface. Also, by the applied enzymatic desizing, starch is removed from the cotton. The cleaning of the cotton surface by washing leads to a more hydrophilic cotton surface, as determined by lower sink in times during the TEGEWA drop test (Table 2). The printing on such clean substrates lead to better adhesion, probable because the better and direct contact between the PLA and the cotton surface. This result of increasing adhesion for washed textile substrate, is determined for the here investigated material combination and no general statement. For other material combinations, also a negative impact of washing on the adhesion could be possible.

In contrast, Mpofu et al. report a different behavior for PLA prints on cotton fabrics, with a decreased adhesion in case of washing the fabric before printing [27]. However, in his study the washing is only performed at $40{ }^{\circ} \mathrm{C}$ and no enzymatic desizing is done. Probable especially the desizing to remove the starch from the cotton surface is an important issue to improve the adhesion of a PLA print. Different pretreatment and cleaning procedures for cotton fabrics before a PLA print are investigated and reported by Kozior et al. [28]. He found a slightly increased adhesion in case of a pretreatment by washing. Unfortunately, no description of the washing procedure, agent, temperature or kind of desizing procedure or agent is given in this reference [28].

If a comparison between the three types of fabrics and their adhesion to PLA has to be made, the ranking is F2 $>$ F4 $>$ F1 (Fig. 9). This ranking is clearly related to the two fabric properties, weight and thickness. A fabric with higher weight and thickness shows better adhesion properties to an applied PLA object compared to fabrics of lower weight and thickness.

Photographs of printed Nylon objects after separation experiments from cotton are compared in Fig. 11. With the
Fig. 10 Photographs of PLA objects on different cotton fabrics after the adhesion test. Compared are grey fabrics and fabrics after washing

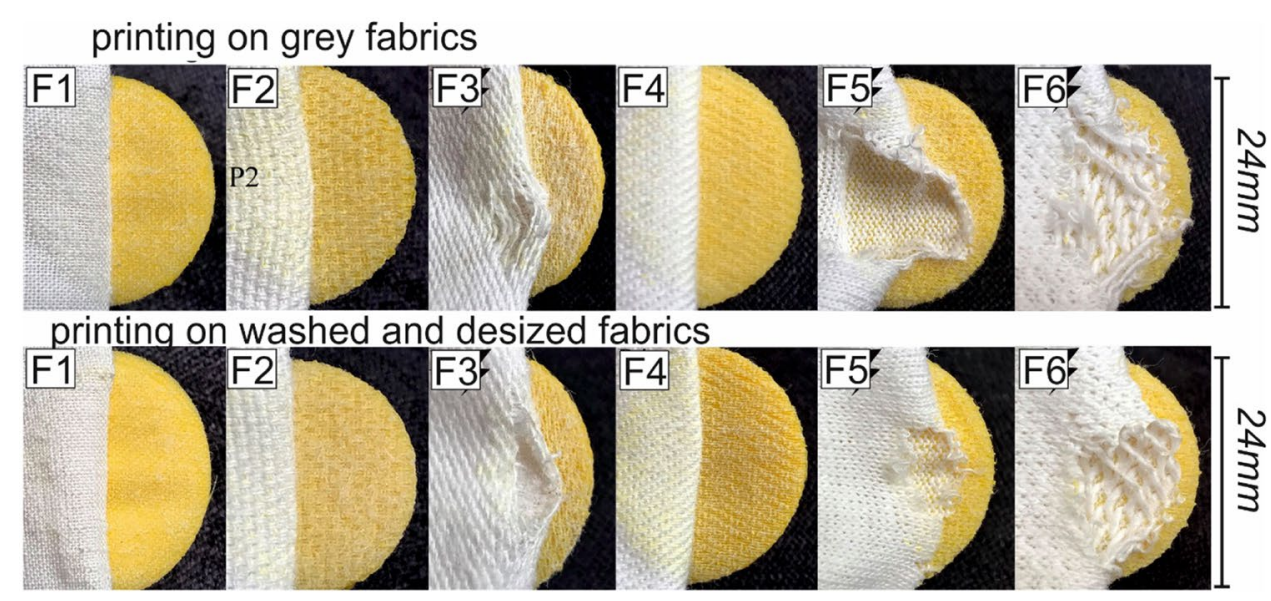


Fig. 11 Photographs of Nylon objects on different cotton fabrics after the adhesion test. Compared are grey fabrics and fabrics after washing

\section{printing on grey fabrics}

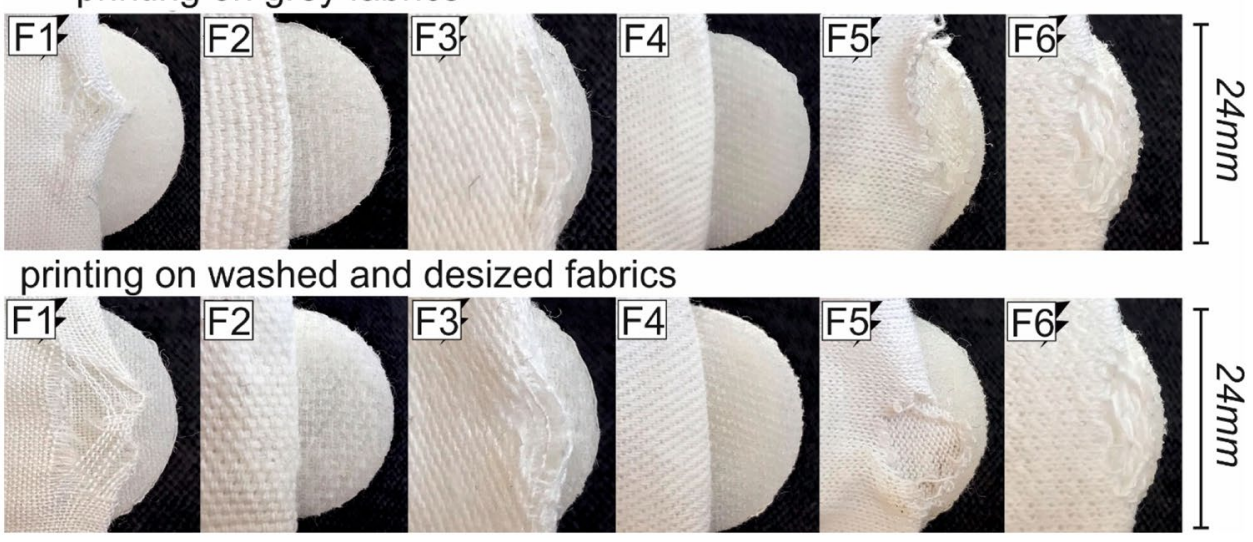

Nylon objects only in case of two cotton fabrics F2 and F4, a separation of Nylon object and cotton is observed without additional damaging of the cotton fabric. The adhesion intensity for Nylon objects can be ranked here F2 $>$ F4, which is the same order as observed for the PLA objects. With higher fabric weight and thickness, the adhesion to the 3D printed Nylon object is increased (Figs. 12 and 13). Also, for the Nylon 3D prints a previous cleaning of the fabric by washing enhances the adhesion to the afterwards printed Nylon object (Figs. 12 and 13). By view on the adhesion results of fabrics F2 and F4, also the performance of PLA and Nylon prints can be compared. For both cotton fabrics, the adhesion to the Nylon print is significantly stronger compared to the applied PLA print. For this result, there are mainly two reasons - one related to process parameters and the other related to the polymer structure. The extrusion temperature

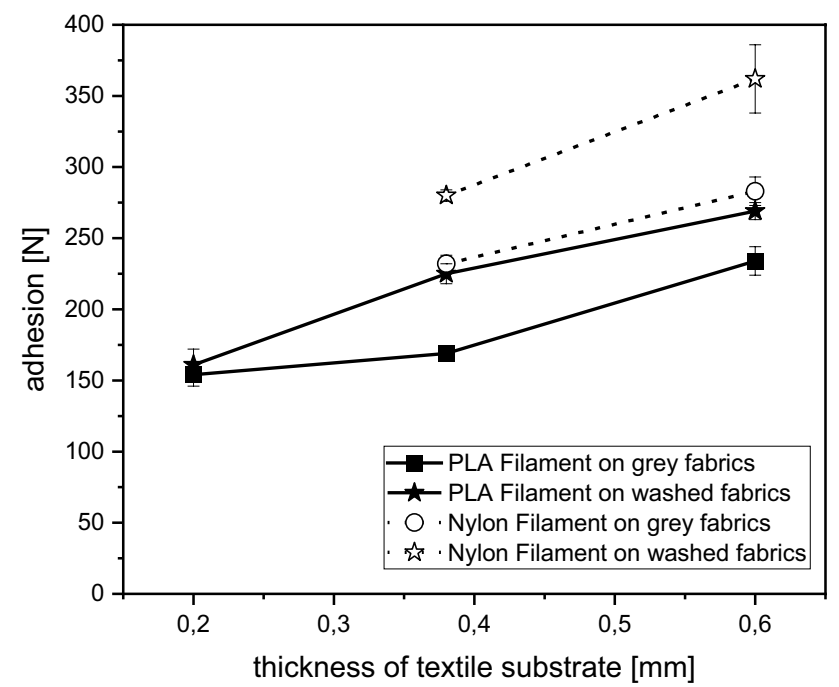

Fig. 12 Adhesion of print on cotton fabrics as function of the thickness of textile substrates. Compared are the measurement data of samples presented in Figs. 10 and 11, where textile samples are not damaged during the adhesion measurement for Nylon is set to $250^{\circ} \mathrm{C}$ and for this higher compared to the extrusion temperature for PLA $\left(220^{\circ} \mathrm{C}\right)$. The higher extrusion temperature for Nylon compared to the temperature for PLA, is related to the higher decomposition temperature $T_{D}$ for Nylon (Nylon $\mathrm{T}_{\mathrm{D}}=390{ }^{\circ} \mathrm{C}$; PLA $\mathrm{T}_{\mathrm{D}}=300^{\circ} \mathrm{C}$ ) [29]. The effect of an increased extrusion temperature leading to an increased adhesion was also described in orientation according to printing parameters where the printing parameters are evaluated. An application at higher temperature can lead to a more fluid polymer melt, which has a better ability to penetrate the structure of the treated cotton fabrics. Additionally, cotton is a cellulosic fiber containing many hydroxy groups and a strong hydrophilicity. The strong hydrophilic properties of the cotton substrates are also documented by the short sink in times determined by the TEGEWA drop test (Table 2). In contrast, PLA is a hydrophobic polymer. Nylon

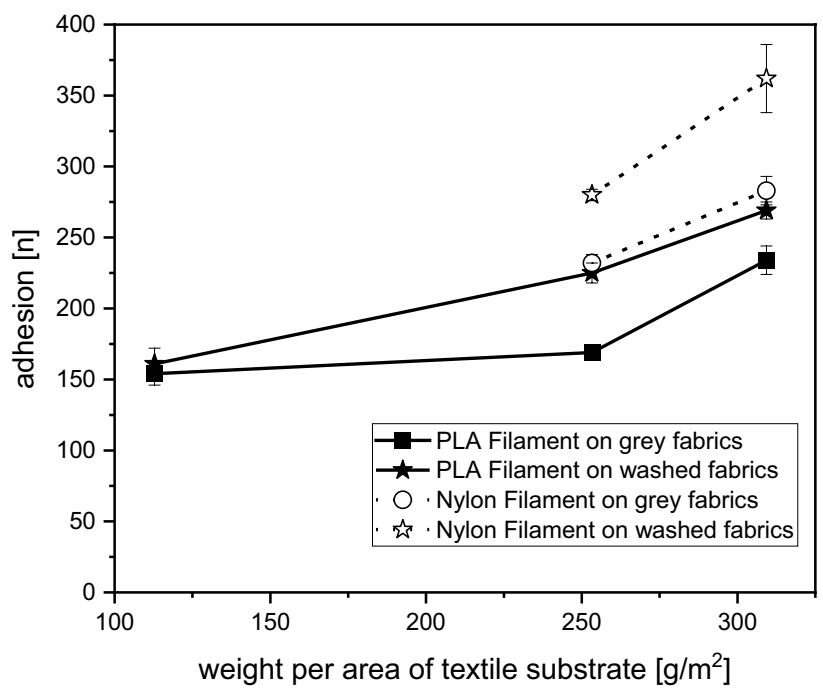

Fig. 13 Adhesion of print on cotton fabrics as function of the weight of textile substrates. Compared are the measurement data of samples presented in Figs. 10 and 11, where textile samples are not damaged during the adhesion measurement 
is less hydrophobic compared to PLA and could have for this reason a better adhesion to the hydrophilic cotton substrate. The different hydrophobicity of both polymers is shown by different contact angle values against water - for Nylon $70^{\circ}$ and for PLA $77^{\circ}[30,31]$. In fact, it can be supposed that a less hydrophobic polymer as Nylon has a higher affinity to the hydrophilic cotton fabric - in comparison to lower adhesion of the hydrophobic PLA print.

\section{Conclusions}

It is possible to apply 3D printed objects on cotton textiles with strong adhesion between both materials. The intensity of adhesion depends on factors from three different categories - the textile properties, the process parameters for printing and the type of printed polymer.

In case of textile material, the adhesion increases with fabric weight per area and thickness. An additional improvement can be achieved, if a precleaning of the textile is performed by washing and desizing. In case of printing process parameters, a strong influence is related to the printing temperatures. Higher process temperatures lead to stronger adhesion. Beside the temperature, also the Z-distance between printing head and table and the printing speed of the first applied layer are important parameters. Z-distance and printing speed should be small enough to reach sufficient adhesion. In case of the material composition of the printed object, it can be assumed that materials which enable higher process temperatures have a certain advantage. Also, the printing on hydrophilic textile substrates like cotton should be done with polymers of lower hydrophobicity.

Finally, it should be stated that the presented comparative study supports a whole view on a broad assemble of different parameters and influences. For this, it can be a helpful tool for practical applications developing 3D printed objects on textile materials made from cotton.

Acknowledgements All product and company names mentioned in this article may be trademarks of their respected owners, even without labelling

Funding Open Access funding enabled and organized by Projekt DEAL.

Open Access This article is licensed under a Creative Commons Attribution 4.0 International License, which permits use, sharing, adaptation, distribution and reproduction in any medium or format, as long as you give appropriate credit to the original author(s) and the source, provide a link to the Creative Commons licence, and indicate if changes were made. The images or other third party material in this article are included in the article's Creative Commons licence, unless indicated otherwise in a credit line to the material. If material is not included in the article's Creative Commons licence and your intended use is not permitted by statutory regulation or exceeds the permitted use, you will need to obtain permission directly from the copyright holder. To view a copy of this licence, visit http://creativecommons.org/licenses/by/4.0/.

\section{References}

1. Liu J, Sun L, Xu W, Wang Q, Yu S, Sun J (2019) Current advances and future perspectives of 3D printing natural-derived biopolymers. Carbohydrate Polym 207:297-316

2. Raddatz L, Austerjost J, Beutel S (2018) 3D-Druck: Chancen, Möglichkeiten, Risiken: Wie eine neue Technologie die Zukunft der Biotechnologie und Chemie prägen könnte. Chem unserer Zeit 52(1):42-50

3. Tsou CH, Yao WH, Wu CS, Tsou CY, Hung WS, Chen JC, Guo J, Wen E, Wang RY, Suen MC, Liu SC, de Guzman MR (2019) Preparation and characterization of renewable composites form Polylactide and Rice husk for 3D printing applications. J Polym Res 26(9):227-236

4. Deleersnyder K, Ruys L (2015) 3D-Druck auf Textilien. Textilplus 3(7/8):23-25

5. Korger M, Lutz M, Rabe M (2015) 3D-Druck kombiniert mit Textil - eine Hybrid-Technologie zur individuellen Erzeugung von Multikomponenten-Textilien. Textilplus 3(11/12):25-28

6. Korger M, Bergschneider J, Neuss J, Lutz M, Mahltig B, Rabe M (2016) Functionalization of textiles using 3D printing - add-onn technology for textile applications testing new material combinations. Vlákna a textil (Fibres and Textiles) 23:106-111

7. Bergschneider J, Korger M, Kyosev Y, Lutz M, Rabe M, Mahltig B (2017) 3D printing for functionalization of textiles - aspects of durability and coating adhesion. Melliand International 22:45-47

8. Sitotaw DB, Ahrendt D, Kyosev Y, Kabish AK (2020) Additive Manufacturing and Textils - State-of-the-Art. Appl Sci 10(5033): 1-21

9. Döpke C, Grimmelsmann N, Ehrmann A (2016) 3D-Druck auf Gestricken. Melliand Textilber 97(4):195-196

10. Korger M, Glogowsky A, Sanduloff S, Steinem C, Huysman S, Horn B, Ernst M, Rabe M (2020) Testing thermoplastic elastomers selected as flexible three-dimensional printing materials for functional garment and technical textile applications. J Eng Fibers Fabrics 15:1-10

11. Grimmelsmann N, Martens Y, Schäl P, Meissner H, Ehrmann A (2016) Mechanical and electrical contacting of electronic components on textiles by 3D printing. Procedia Technol 26:66-71

12. Zhang $M$, Zhao M, Jian M, Wang C, Yu A, Yin Z, Liang X, Wang H, Xia K, Liang X, Zhai J, Zhang Y (2019) Printable Smart Pattern for Multifunctional Energy-Management E-Textile. Matter $1: 168-179$

13. Eutionnat-Diffo PA, Chen Y, Guan J, Cayla A, Campagne C, Zeng X, Nierstrasz Y (2019) Stress, strain and deformation of poly-lactic acid filament deposited onto polyethylene terephthalate woven fabric through 3D printing process. Sci Rep 9(14333):1-18

14. Sanatgar RH, Cayla A, Campagne C, Nierstrasz V (2019) Morphological and electrical characterization of conductive polylactic acid based nanocomposite before and after FDM 3D printing. J Appl Polym Sci 47040:1-11

15. Graßmann $C$, Grethe $T$, van Langenhove L, Schwarz-Pfeiffer A (2019) Digital printing of electroluminescent devices on textile substrates. J Eng Fibers Fabrics 14:1-10

16. Narula A, Pastore CM, Schmelzeisen D, El Basri S, Schenk J, Shajoo S (2018) Effect of knit and print parameters on peel strength of hybrid 3-D printed textiles. J Textiles Fibrous Mater $1: 1-10$

17. Unger L, Scheideler M, Meyer P, Harland J, Görzen A, Wortmann M, Dreyer A, Ehrmann A (2018) Increasing Adhesion of 3D Printing on Textile Fabrics by Polymer Coating. Tekstilec 61(4):265-271

18. Meyer P, Döpke C, Ehrmann A (2019) Improving adhesion of three-dimensional printed objects on textile fabrics by polymer coating. J Eng Fibers Fabrics 14:1-7 
19. Spahiu T, Al-Arabiyat M, Martens Y, Ehrmann A, Piperi E, Shehi E (2019) Adhesion of 3D printing polymers on textile fabrics for garment production. IOP Conf Series: Mater Sci Eng 459(012065):1-6

20. Mpofu NS, Mwasiagi JI, Nkiwane L, Njuguna D (2019) Use of regression to study the effect of fabric parameters on the adhesion of 3D printed PLA polymer onto woven fabrics. Fashion and Textiles 6(24):1-12

21. Sanatgar RH, Campagne C, Nierstrasz V (2017) Investigation of the adhesion properties of direct 3D printing of polymers and nanocomposites on textiles: Effect of FDM printing process parameters. Appl Surf Sci 403:551-563

22. Kozior T, Blachowicz T, Ehrmann A (2020) Adhesion of threedimensional printing on textile fabrics: Inspiration from and for other research areas. J Eng Fibers Fabrics 15:1-6

23. Han Y, Kim J (2019) Study on Peel Strength Measurement of 3D Printing Composite Fabric by Using FDM. J Fas Bus 23(2):77-88

24. Wurster P, Schmidt G (1987) The TEGEWA drop test, a method for the rapid determination of textile fabric absorption. Melliand Textilber 68:581-583

25. Bahners T (2011) The do's and don'ts of wettability characterization in textiles. J Ads Sci Technol 25(16):2005-2021
26. Malengier B, Hertleer C, Cardon L, van Langenhove L (2017) ITMC2017 - International Conference on Intelligent Textiles and Mass Customisation

27. Mpofu NS, Mwasiagi JI, Nkiwane LC, Githinji DN (2020) The use of statistical techniques to study the machine parameters affecting the properties of 3D printed cotton/polylactic acid fabrics. J Eng Fibers Fabrics 15:1-10

28. Kozior T, Döpke C, Grimmelsmann N, Junger IJ, Ehrmann A (2018) Influence of fabric pretreatment on adhesion of three-dimensional printed material on textile substrates. Adv Mechanical Eng 10(8):1-8

29. Wojtyla S, Klama P, Baran T (2017) Is 3D printing safe? Analysis of the thermal treatment of thermoplastics: ABS, PLA, PET, and nylon. J Occup Environ Hyg 14(6):D80-D85

30. Ellison AH, Zisman WA (1954) Wettability studies of Nylon, polyethylene terephthalate and polystyrene. J Phys Chem 58(6):503-506

31. Thanki PN, Dellacherie E, Six JL (2006) Surface characteristics of PLA and PLGA films. Appl Surf Sci 253:2758-2764

Publisher's Note Springer Nature remains neutral with regard to jurisdictional claims in published maps and institutional affiliations. 